Article

\title{
Portraying Reality: Perceptions and Realities of Youth in Korean Theatre for Adolescents
}

\author{
Joohee Park ${ }^{1, *}$ \\ 1 Adjunct Professor, Department of Theater and Film, School of Performing Arts, Kookmin University, 77 \\ Jeongneungro, Seongbuk-gu, Seoul, 02707 Korea \\ * Correspondence: jooheepark@kookmin.ac.kr; Tel.: +82-2-910-4466
}

\begin{abstract}
In this paper I analyze the three plays produced during the inaugural season (2011) of the Theatre for Young Audiences Research Center of the National Theater Company of Korea and place it within the context of contemporary ideas and realities surrounding Korean youth. In the first part of this paper I explore how Korean youth are perceived by society and the reality in which they live. In the second part I analyze the aforementioned plays, especially through the directions of the production and portrayal of adult and young characters. The last part offers concluding thoughts. Through this study I ask the question of whether if it is possible for theatre to actually portray the realities of adolescents, when in fact the identities of youth are still fluid and the experiences of youth as diverse as those of adults. I suggest that theatre artists break free to depict reality or educate audiences and focus on providing a critical experience to adolescents.
\end{abstract}

Keywords: theatre for adolescents; theatre for young audiences; art for youth

\section{Introduction}

A few years ago I had an interesting conversation with a researcher at the National Theater Company of Korea. We were discussing the Korean film "Hwhy," in which a seventeen year old boy realizes that the five men who had raised him (and called themselves his fathers) were in fact his kidnappers. Full of rage, the boy explodes into a killing spree, employing homicidal methods his fathers had taught him themselves. The researcher commented that the protagonist. Hwhy, could be viewed as a contemporary hero to teenagers, as he takes matters into his own hands to fix the absurd reality of adults. I wryly informed the researcher that despite the fact that the film is about a seventeen year old, and that that actor playing Hwhy was sixteen years old at the time of filming, the film was rated " $\mathrm{R}$ " and therefore off limits for teenagers under eighteen years of age. An interesting conversation followed, as we discussed which part of the film had earned its rating: a hint of sexual content? Gun violence? Frequent swearing? Video games and television shows popular among teenagers are often just as, if not more, violent than the film discussed. Popular culture is inundated with sexual content, frequently carried out by underage pop stars. However the Korean Ministry of Culture had deemed "Hwhy," a film about a teenager who solves adult-made problems with methods taught to him by adults, unsuitable for teenagers to watch. Questions regarding appropriate teenage characters for teenage viewers began to rise. We asked ourselves, how are youth portrayed in art, and what do those portrayals signify? As a researcher primarily working in the field of Theatre for Young Audiences (TYA; theatre for children and adolescents produced by professional theatre makers), I became intrigued to investigate how youth are portrayed in Korean TYA and how those portrayals relate to the perceptions and realities surrounding youth.

Koreans call Theatre for Young Audiences "Theatre for Children and Adolescents [Adong Cheongsonyeon Yeongeuk]," differentiating between children and adolescents. While theatre for children is an active field in Korea, theatre for adolescent aged youth is scarce. For example, $21 \%$ of all theatre performances in Korea during the year of 2010 were TYA productions, but among those 530 plays less than $10 \%$ were Theatre for Adolescents (ARKO 2010). Therefore the inauguration of the TYA 
Research Center of the National Theatre Company of Korea in 2011 marked a significant event in Korean TYA, as it has thus far focused on producing plays for adolescent youth. The three plays in the inaugural season, The Boy Did It [Sonyeone Geuraetda]', The Wrestling Season, and The Red Bus [Bbalgan Bus], each deal with juvenile crime, identity and bullying, and teenage single-motherhood, respectively. Considering the fact that the National Theater Company of Korea is a government sponsored institution, the impact these productions play in Korean TYA is quite substantial. Although reviews have been published on these plays, there are few researchers investigating TYA from a scholarly perspective in Korea, and therefore this study is, to my knowledge, the first research paper that analyzes the inaugural season of the TYA Research Center.

In this paper I analyze the three plays produced during the inaugural season (2011) of the Theatre for Young Audiences Research Center of the National Theater Company of Korea (hereafter the TYA Research Center) and place it within the context of contemporary ideas and realities surrounding Korean youth. In the first part of this paper I explore how Korean youth are perceived by society and the reality in which they live. In the second part I analyze the aforementioned plays, especially through the directions of the production and portrayal of adult and young characters. The last part offers concluding thoughts. Through this study I continuously ask the question of whether art must take responsibility in depicting the reality of this world, and, if so, how? Especially in the context of art for youth, must art for youth bear a heavier responsibility than art for adults?

To answer these questions I use my own analysis of the plays both in script form and stage version, evaluate audience response forms obtained from the TYA Research Center, and consult previous literature on Korean youth and art for youth. My theoretical framework is informed by Louis Althusser's concept of Ideological State Apparatus, in which cultural institutions such as theatre are used by the dominating class to indoctrinate members of the society with the preferences, values, and desires of the ruling class (Gramsci 1971). Therefore TYA reflects the hegemony of the ruling class, which is not only a class issue but also a generation issue in this case, as the ruling class is most always consisted of adults. Therefore TYA will always reflect what adults believe is good for youth, rather than what youth want or take pleasure in. Hence analyzing art for youth provides an insight into what is perceived of youth by society.

\section{Realities of and Art for Adolescents in Korea}

\subsection{Adolescence in Korea}

While grade school age children are considered to be dependent on their guardians, adolescents are defined as "children who are growing independent" (Park and Yu 2011) and who have "started to form self-consciousness but are still dependent on their parents or other guardians" (Kim 2013). Jennifer Chapman states that adolescence is "a historically specific maturation period identified by developmental and social psychology, as well as psychoanalysis" (Chapman 2005). Such literature suggests that adolescents are undergoing a different stage than younger children and therefore are entitled to a different set of rights and rules. However definition of what age marks adolescence is not easy to define in Korea, as even judicial language is not consistent on the boundaries of "adolescence." For example, A Korean youth must be over thirteen years of age to hold a part time job, but must be over fourteen years old to be tried as an "adolescent" in a trial. The law states that a person over eighteen years of age can be drafted to the military of get married, which means that a person over eighteen is considered an "adult." However one must be nineteen years old to watch an " $R$ " rated film or cast a vote in the elections. A person must be twenty years old to buy alcohol or tobacco in Korea, and to register as a candidate for political office one must be at least twenty-five years of age. Such inconsistencies make it almost impossible to define legally which years mark a youth's adolescence in Korea. Culturally however, Koreans generally consider youth between the ages of thirteen (first year of middle school) and eighteen (last year of high school) as adolescents, and widely use the term " 1318 " to indicate this period of life. 
Adolescents in Korea are often forced to endure long school hours and devote after school hours to studying. According to statistics published by the Ministry of Education in 2013, about 1.1\% of youth between the ages of seven and eighteen do not attend school (KME 2013); therefore it is safe to assume that in Korea most adolescents are students. Middle and high school students in Korea are expected to dedicate their time and effort to preparations for their college entrance exam. Gaining admission to a prestigious college is considered the prime goal of every student, and adolescents are often told that their desires to enjoy life can wait until they are college students. In order to gain admission to a good university one must do consistently well on exams throughout high school and also earn good marks in the yearly National Academic Aptitude Test. Held every November, flights and construction activities are help up during test hours to allow students to concentrate on the exam, and police escort those who are late for the exams in their squad cars. Such measures may seem trivial, but they testify just how dedicated Koreans are towards college admission. An adolescent studying for college entrance exams is granted abundant generosity and protection. However, once an adolescent decides to discontinue studies or move outside the boundaries of social norm, that person is immediately labeled as "problematic" and renounced, as s/he may influence other students to act similarly. Sociologist Yoo-seong Cheong criticizes that in Korea there is no adolescent culture but only school culture, as "adolescents are only allowed to be students and nothing else" (Cheong 1997). The period of adolescence in Korea according to society, then, is not one of self-exploration or development of independence but a grace period until life truly starts in college. In other words, society offers protection through regulation to adolescents if they are willing to stay within perceived boundaries and expectations. A quick search on academic journal articles regarding adolescents are focused on either problems regarding adolescents (i.e. teen drinking, teen smoking, teen delinquency) or policies regarding adolescents (i.e. art education policy, teen medical policy). Studies regarding the cultures and desires of adolescents are visibly absent, only appearing in non-academic journals. This absence reflects Korean society's attitude towards adolescents, treating them as objects of protection and/or punishment and not as individuals with preferences and desires.

\subsection{Theatre for Adolescents and the TYA Research Center's Inaugural Season}

As adoelscents are overwhelmed with school work and afterschool lessons, secondary school students have little time to spare for extracurricular activities. Even when they have time for entertainment few adolescents attend live theatre regularly, preferring to play video games or watch films instead. The governent states that it is putting efforts into encouraging cultural and artistic activities among youth, but scholars such as Jin-sook Lee argue that such efforts are not based on the realities of adolescents' lives and therefore wasted (Lee 2012). Few adolescents are given the option to actively participate in artistic activities unless they seek such events privately outsife of school walls. Because of such issues, theatrical productions for adolescents remained scarce in Korea before 2011, with only the theatre troupes Hakchon and Jeendong producing professional Theatre for Adolescents regularly. The former mostly focused on issues teens faced in school while the latter produced plays that dealt with love and sexuality of adolescents (Kim 2011). Though the work of the two companies were generally well received, they suffered financial difficulty because of poor attendance rates and lack of public funding (Park 2010).

When the TYA Research Center was founded in 2011, administrators had not planned for it to focus its entire season on Theatre for Adolescents. However the pressing need for art for adolescents in contrast to the relative abundance of art for children became the vehicle behind the TYA Research Center's concentration on older youth. The TYA Research Center ambitiously set out to introduce productions that young audiences can relate to in content and aesthetics. Y.A. Choi, then-head of the TYA Research Center, states that "we do not want to make Theatre for Adolescents that adults want us to make. We will not percieve from the adult's point of view but level with the adolescents' points of view" (Park et al. 2013). Choi seems to be referring to the fact that TYA is often made to please adult audiences, whether they be parents, teachers, or critics. This is because despite the fact that the 
TYA is made for young audiences, the people who are paying for the productions are most likely adults. One of the reasons why the aforementioned theatre troupe Jeendong suffered financial hardship is because they refused to fulfill adults' expectations on what art for adolescents ought to be and produced plays dealing with issues such as teenage pregnancy. This resulted in schools cancelling tour bookings. Therefore the decision by the TYA Research Center to stick to the adolescents' point of view stands out as a significant one and brings up the question of whether it was possible only because of reliable government funding. Such point should be explored in a separate future study.

The first play produced by the TYA Research Center was The Boy Did It (hereafter The Boy), adapted from the Australian play The Stones. The director I. Nam states that she came to select the play after reading a news article about an innocent pedestrian being hit and killed by a stone that a child threw from the fifteenth floor of a residential building in Gwangju, Korea, and how the community was buzzing about who is to blame in this case (Nam 2013). Rather than using the original script the producers of The Boy asked Korean playwright H. Hwang to rewrite and adapt the story of The Stones so that it could speak closer to a Korean audience. In The Boy, fourteen year old Sangsik and fifteen year old Minjae hang out in a neighborhood that is no longer occupied. They talk about recent experiences of being bullied by schoolmate Doeji (meaning "pig" in Korean) and decide to throw stones at Doeji from a bridge when Doeji passes by on a motorcycle. One of the stones they throw end up hitting a car and results in a fatal accident. The two boys run away and hide in their homes but eventually they are caught and investigated by two police officers. At the end, both are released on probation and come face to face with the widow of the deceased driver. The play ends with the usually running Sangsik and Minjae walking back and forth, not knowing what to do, finally slowing down to a stop.

The second play in the inaugural season's lineup was The Wrestling Season, originally written by US playwright Laurie Brooks and adapted slightly by Hyeonji Hwang (same playwright that adapted The Boy). In the play, eight high school students struggle with issues of identity, rumors, and peer pressure. Mingi and Gangseok are best friends and both on the school wrestling team. Their rivals Gitae and Yeongpil start whispering that Mingi and Gangseok are in a gay relationship and the rumor quickly spreads throughout the school. To put down such rumors Mingi asks Jua, a girl rumored to be promiscuous, to go out, which she accepts. When Mingi does not make physical advancements towards Jua after a few weeks of dating, a rumor spreads again that he is actually using Jua to hide his sexual orientation, which causes Mingi to try to force Jua into a sexual relationship. Jua breaks up with Mingi, saying she is disappointed in him. Meanwhile, Gangseok is beat up by anonymous bullies the day before an important wrestling match and decides to quit the team. Mingi finds Gangseok hiding away and convinces him to enter the match. Gangseok loses to his rival Yeongpil at the match while Mingi wins against his rival Gitae. The play ends with another rumor being started among students regarding the sexual relationships between Yeongpil, his girlfriend Hyeri, and other female students. After the play is over, audience members are invited to participate in a Boal-esque forum with the actors who played the students still in character, and the actor who played the referee stepping in as facilitator.

The world premiere of The Red Bus was the last production of the TYA Research Center's inaugural season. The play was written and directed by Geunhyeong Park, a well-known theatre artist in Korea. The protagonist of The Red Bus is Sejin, who from first impression seems like an ordinary high school student. She is part of a singing trip which she formed with her best friends, Haneul and Jeongmin. Sejin's music teacher from school is infatuated with her, calling her late at night and leaving numerous messages. Sejin seems to be constantly in need of money, and her friends suspect that she is keeping a secret but cannot figure out what it is. Sejin's parents had seperated when she was younger and she had been living alone for the past few years, with receiving only minimal financial assistance from her parents. When Sejin is caught stealing baby formula it is 
revealed that she has a baby, whom she has been raising alone with the help of a kind neighbor. Sejin absolutely refuses to reveal the identity of the baby's father to anyone, only saying that he was much older than she is and that they are no longer in a relationship. The teachers discuss what they should do about Sejin, some stating that she should be expelled for having sexual relations. When Sejin's mother appears for the first time, she tries to persuade her daughter to put the baby up for adoption. She also futilely probes about the identity of the father. Sejin, in a long monologue, vents how everyone asks her about the father while nobody asks about the baby. She states that she borrowed money from her friends and gave birth to the baby alone at a seaside resort, and that she has been taking care of the baby herself. She asks her mother for one thing only, which is convincing her teachers to let her stay in school. In the next scene, Sejin tells her friend Dongwon that she is quitting school because everyone seems uncomfortable with her being in the classroom. And that she will now focus on making a living. As Sejin exits the stage the sound of car brakes are heard and the play ends with Sejin's classmates attending her funeral while singing the song Sejin wrote for her trio.

The three plays produced in the inaugural season of the TYA Research Center were generally well received by both critics and the public. For instance, theatre critic Kim asserted that all plays produced by the TYA Research Center were of "stable" quality and that the productions contributed to the field of Theatre for adolescents (Kim 2014). All three plays were revived at the National Theater Company in Seoul in 2013, and The Boy Did It toured eleven cities around Korea in 2012. In the next part of this study, I analyze how the three plays reflect or reject the realities and society's perceptions of youth by examining the aesthetics and portrayals of characters in the three productions. Cultural theorists such as Louis Althusser and Antonio Gramsci state that cultural institutions such as theatre help indoctrinate members of society to the ideas of the ruling class (Althusser 1971; Gramsci 1971). This statement is often even more legitimate in TYA compared to theatre for adults because theatre for young audiences is made by a social demographic who, by default, have more power than their audiences. This is why, as mentioned in a previous section, TYA frequently conforms to the ideas and perceptions of adults. However in this paper I explore TYA that was made by adults who state that they want to give agency to youth and that they do not wish to nor need to please adults. In this paper I explore how the lives of youth are portrayed in theatre produced under such unusual circumstances and further question if the realities of youth can actually be sufficiently depicted in art made by adults.

The Boy Did It and The Wrestling Season were written by foreigners but were adapted by a Korean playwright and The Red Bus was written by a Korean playwright. In all three cases the creators seem to have put extra effort into making the language and settings familiar to Korean youth. The foreign names of characters in The Stones and Brook's The Wrestling Season were changed to Korean names. Considering that B. Brecht used foreign sounding names for his characters to alienate audiences, adapting the original names to Korean sounding ones is an essential step in allowing audiences to emphathize with characters. One thing I noticed when reviewing audience response sheets completed by adolescents who watched the plays were the freuqent comments on the "bad words" used in all three productions. The TYA Research Center organized a "teenage supporters group" for each play during its rehearsal period for feedback, and the group shared their opinions on various elements of the play before the premiere. As a result, all three plays employed slang and curse words commonly used by Korean youth. However, the use of swearing resulted in mixed responses from the audience. While some wrote that "bad language" made the play more enjoyable or more familiar, just as many responses stated that they were bothered and/or alienated by the language. A few wrote that the curse words used by the characters were out of date and no longer used commonly. This brings up an interesting point; while the people creating the plays relied on the advice of youth to reflect the "real language" of that generation, some youth actually found that language to be alienting. The audience responses reminds one that adolescents come from diverse cultures and spectrums of life and that they do not necessarily employ a uniform style of language. 
Music and movement played an essential role in all three plays discussed in this paper. In The Boy, the theatre was set up that audiences sat in two sides facing each other and the actors moved both in between and around the audiences. A live rock and roll band played throughout the play from one corner of the theatre, and Minjae and Sangsik never stopped moving until the very end, giving a deep sense of unrest and fast paced heartbeat. In The Wrestling Season, the eight actors playing high school students actually wrestle each other during the entire production. The use of wrestling is important in this play, as it is not only the material of the play but also because the activity serves as an analogy of the various relationships between the characters. The referee occasionally steps in to stop or judge a move, even when characters are engaged in non-wrestling related conflicts. In The Red Bus Sejin is part of a singing ensemble that write their own songs. Sejin and her friends claim that writing songs and singing them is the only means through which they can express their true feelings. Sejin has a male friend, Dongwon, whom she hangs out with occasionally in the park. The two of them take turns riding his bicycle on stage, and it is the only time Sejin appears carefree. It is interesting that, while the three plays use entirely different movements and music to portray the worlds of the three stories, the young characters are all continuously moving actively. Such physical activity gives an aura of energy but also of restlessness and unease.

In terms of characters, The Boy Did It, The Red Bus, and The Wrestling Season portray adult and younger generations in a different manner. There are two types of adult characters in The Boy: the two police officers and the parents of Sangsik and Minjae. The former are played by the same actors who play Sangsik and Minjae and the latter do not appear on stage but are talked of by different characters. While the police officers are responsible and caring in their own ways, the parents are illustrated as rather useless. Minjae's mother is obsessed with religion and begs God to punish then forgive her child when she finds out about the crime. It is hinted that Minjae's father is an oppressive and violent figure in the family. Sansik lives with his mother who is never home, busy making a living, and she lies to the judge that Sangsik had never made trouble before. The issue of age is important in this play, as Sangsik, being fourteen years old, is entitled to be tried as a child while Minjae, fifteen, is tried as an adolescent. Adults are obessessed about this difference in this play, as well as who is to blame for this accident, when in reality the two friends were just playing together and were equally at fault. This element accentuates the dissonance between the adults' perception and the reality of the situation.

In The Wrestling Season there is only one adult character, the referee. As metioned before, he steps in during the play to stop a conflict or judge a move between the characters, but otherwise keeps his distance. This can be seen as adults giving youth space to explore their identities and solve their own problems. However when events turn violent, such as when Mingi tries to rape Jua or Gangseok is beat up by bullies, the referee only watches and makes no move to stop the action. This is problematic from the young character's points of view and perhaps exemplifies how adults seem to step in at inapproriate times and are absent when actually needed.

Basically all adult characters are irresponsible and only bring misery to youth in The Red Bus. Sejin's parents are absent from her life; they are seperated and only send her a monthly check without even checking on her periodically. They do not even realize that Sejin has given birth to a child months ago until infromed by a school teacher. Even after she finds out about the situation, Sejin's mother does not offer help, she apologizes for her absent then justifies her action by saying she is content with her current relationship and life. Haneul's parents are in an unhealthy relationship, with her mother only interested in her hobby (hiking) and her father recently having been caught cheating with another woman. Haneul confesses that despite the fact that she lives with both of her parents neither is interested in whether she eats properly or goes to school. The teachers at Sejin's school are just as irresponsible: The music teacher declares his love for Sejin and tries to kiss her after continuously seeking her throughout the play; Ms.Kang, another teacher at Sejin's school, openly states to other teachers that she has no interest in her students and that she is only interested in the 
well-being of her daughter and her monthly paycheck. She strongly argues that Sejin should drop out of school as Sejin has "crossed the line," from the side where school and society need to protect a child to the other side where a child no longer deserves protection. This statement reflects the contemporary Korean society's perception that adolescents deserve protection only as long as they stay within the prescribed boundaries. Once one crosses that line one must be pushed out and seperated from those who are still being protected. Ms. Kang's point is a common view in Korean society yet ironic as Sejin was never sufficiently protected by adults even before she became pregnant, yet the blame is put on her and her only. Legally Korean adolescents who are pregnant or have children (evidence of sexual experience) are entitled to stay in school; however in reality it is extremely difficult for such youth to attend school because of social stigma.

Why are adults unanimously portrayed as absent and/or negative influences on the young characters in all three plays? Does it reflect what adults (including the creators of the play) think how adolescents percieve the older generation, or is it admittance that adults can be powerless in solving problems regarding youth? Whatever the reason the group of adults in The Boy, The Wrestling Season, and The Red Bus offer little consolation or help towards the protagonists of the three plays. There are also no adults that stand as mediator or in-between figure between the adults and youth. Adults in Korea often complain that younger generations use "alien language" with their slangs and abbreviations; often times older and younger generations have a hard time communicating with each other even though they supposedly speak the same language. The three plays seem to accentuate such discord and severance between the two generations by contrasting the two groups in language, movement, and thought.

The ending of the three plays were quite different from each other but nevertheless reflected how Korean society treats youth. The ending of The Boy is somewhat open: the two boys stop running and face each other from opposite sides of the divided stage. "We may not have a reason to run anymore," Minjae says. "Or we may make up a reason to run even faster," says Sangsik (Hwang 2011). Running in this play symbolizes youthfulness and livelihood of the two boys. The unexpected events forced the two youth, still only fifteen and sixteen years old, to grow up and stop running.

The ending of The Wrestling Season was intriguing as it was accompanied by an Augusto Boal style forum. While the story itself ended with new rumors starting to spread after the big match, audience members were invited to participate in a twenty-minute forum immediately following the play. In that forum, the actor who played the referee stepped in as facilitator and encouraged audience members to ask questions or say something to the characters, and also line up the eight characters in order of "innocence" in spreading rumors. The dramaturg of The Wrestling Season writes that the success of the pay depended on how well the forum proceeded after each production (Kim 2013). Audience responses varied on the event, however, with some stating that the forum was fun and allowed them to think more deeply about the issues, while others writing that they felt like they were in an ethics class. Seong-hee Jang, a critic and playwright, claims that she felt "cheated" by the forum because it "just felt too educational" (Jang 2013). The orignial play by Brooks comes with a forum section but it could have been easily removed during the adaptation process. The fact that the creators of the plays found it important to have young audiences sit down after the play and talk about what they saw seems to reflect society's perception that art for youth must be enlightening.

Perhaps the play with the most controversial ending, however, is The Red Bus. The death of Sejin shocked many members of the audience and was one of the most talked about issue in the audience response sheets. Most who commented on the ending wrote that they felt unhappy about Sejin's death, as it seemed to indicate there is no hope for a young person in Sejin's situation. In a printed interview, playwright and director of The Red Bus Geunhyeong Park says that he had no choice but to kill Sejin because "adults are the problems that young people are facing. ... Youth are actually dying because of adults. ... We all have our secret babies, and nobody can take care of that baby. A student who had a baby cannot survive" (Park et al. 2013). That is to say, Sejin's baby is an analogy 
of deep secretive problems everyone has, and the cause of such problems lie in adults. Sejin's death by accident is actually not a literal accident but a death that was prone to happen in one way or another, given her circumstances. The ending of The Red Bus is on one hand quite credible and reflects the reality of Korean youth. On the other hand, however, it can, if inadvertently, send out a message to young audiences to stay within the protected boundaries and not become like Sejin. If the message that the playwright/director of The Red Bus wants to declare is that adults are the problems of youth and that young people are dying because of adults, then perhaps this play should not be a Theatre for Young Audiences production but a play for adults.

\section{Discussion}

The inaugural season of the TYA Research Center was a significant event in the history of theatre in Korea. It brought new discourse to the genre because of three main reasons: First, it was the first time National Theater Company or any other government funded theatre institution produced a full season of theatre for adolescents. Such support brought not only financial stability to explore new artistic turf but also drew in well-known theatre artists who had previously not worked in TYA. Second, operating a "teenage supporters group" to provide feedback for productions was, and still is, an unusual experiment. Most TYA practitioners admit to their limitations in understanding the lives of their audiences and attempt to lessen that gap through research and reflections on past experience. However the artists and producers at the TYA Research Center took a further step to invite target audiences to advise artists to make the productions more realistic for adolescents. Third, because all three shows in the inaugural season were performed at the National Theater Company's theatre spaces, numerous schools decided to bring groups of students to the theatre. Such action is a rare occurrence and was only possible because of the label that "The National Theater Company" was producing the shows. The foundation of the TYA Research Center and its inaugural season therefore transformed the practices of not only Theatre for Adolescents but also theatre in general.

As mentioned in previous sections, the three plays analyzed in this study offer an insight into what adults think youth want in art, or what they perceive as the realities of youth. Unlike most previous Theatre for Adolescents productions staged in Korea, the staging steps away from the classroom and actively employs the language of contemporary youth. However, during the process of research and analyses, I became skeptical of whether it is even possible for Theatre for Adolescents to portray the realities youth are facing. This is not necessarily because of the lack of effort or the generation gap, but because the realities and experiences of youth are not homogenous. Korean adolescents may share some similar experiences, but they are diverse in class, sexual orientation, sexuality, age, school grade, and many more elements. In addition to that, adolescence is a period of self-exploration and development, during which identities are fluid. Therefore it is extremely difficult to create a character teenage audiences can broadly identify with. What should, then, theatre for adolescents aim for? I believe that, like any other theatre production, theatre for adolescents should aim for pleasure, room for imagination, and critical discourse. This does not necessarily require realistic portrayals of characters or after-show forums but well thought out art. Of course I am aware of the fact that such statement is idealistic and not necessarily a new idea, but I find it worth pointing out once more in this rare academic article on Korean Theatre for Young Audiences.

Throughout the play The Wrestling Season, characters continuously say, "You think you know me, but you don't" (Brooks 2010). This statement seems to come as a declaration to adults who are making art for youth: you think you know youth, but you don't. Young audiences are fluid in identity and come with diverse experiences; the reality of one youth may come as a fantasy to another.

Acknowledgments: This work was supported by the National Research Foundation of Korea Grant funded by the Korean Government (NRF-2014S1A5B5A07041644).

Conflicts of Interest: The author declares no conflict of interest.

References 
(Althusser 1971) Althusser, Louis. 1971. Lenin and Philosophy and Other Essays. New York: Monthly Review, pp.142-43. 978-1583670392.

(ARKO 2010) Art Council Korea. 2010. Culture and Arts Yearbook. Seoul: Arko, pp. 234-235.

(Audience Responses 2011-2013). Audience Responses. TYA Research Center of the National Theatre Company of Korea. Unpublished.

(Brooks 2000) Brooks, Lauri. 2000. The Wrestling Season. New York: The Dramatic Publishing Company. 9781583420331

(Chapman 2005) Chapman, Jennifer, 2005. The Theatre Kids: Heteronormativity and High School Theatre. Ph.D. Dissertation, University of Wisconsin, Madison, USA.

(Cheong 1997) Cheong, Yoo-seong. 1997. Neogeureo-I Yongseoharyeomuna. Saerosseuneun Cheongsonueon Iyagi. Edited by Ddo Hanaeui Munhwa. Seoul: Ddo Hanaeui Munhwa, 15-25. 8985635271

(Gramsci 1971) Gramsci, Antonio. 1971. Selections from Prison Notebooks. Ed. and Trans. by Quintin Hoare and Jefferey Nowell-Smith. London: Lawrence and Wishart, pp.181-82. 978-0717803972.

(Hwang 2011) Hwang, Hyeon-ju. 2011 Sonyeoni Geuretda. Unpublished play.

(Jang 2013) Jang, Seong-hee. 2013. Three Years of Experiments and Issues at the National Theater Company's Theatre for Adolescents. Paper presented at Iyagipan, Seoul, 11 May 2013.

(Kim 2013) Kim, Okran. 2013. You Think You Know Me, But You Don't. Yeongeuksokui Cheongsonyeon, Cheongsonyeonsokui Yeongeuk. Edited by TYA Research Center. Seoul: NTCK, 87-97. 979-11-85389-02-8.

(Kim 2011) Kim, Yumi. 2011. The Difficulty of Theatre for Adolescents. The Korean Theatre Journal 62: 35-39.

(Kim 2014) Kim, Yumi. 2014. The Surge in Theatre for Adolescents, Is it Growth of Theatre for Adolescents. The Korean Theatre Journal 73: 27-30.

(KME 2013) Korea Ministry of Education. 2013. Investigations of Discontinuation of Education Among Elementary, Middle, and High School Students. Korea Ministry of Education Press Release.

(Lee 2012) Lee, Jin-sook. 2012. A Qualitative Inquiry on the Perception and Experience of Culture, Art, Sport Acitivities in Adolescents. Studies on Adolescents 19: 345-365.

(Park et al. 2013) Park, Geun-hyeong, Woo Yeon, Lee Sook-gyeong, and Minwoo Choi. 2013. Ddo Hanaeui Cheongsonyeongeukeul Malhada. Yeongeuksokui Cheongsonyeon, Cheongsonyeonsokui Yeongeuk. Edited by TYA Research Center. Seoul: NTCK, 117-129. 979-11-85389-02-8.

(Park 2013) Park, Geun-hyeong. 2013. Bbalgan Bus. Yeongeuksokui Cheongsonyeon, Cheongsonyeonsokui Yeongeuk. Edited by TYA Research Center. Seoul: NTCK, 214-162. 979-11-85389-02-8.

(Park 2010) Park, Joohee, 2010. A Decade of Transformation: A Critical View of Theatre for Children and Young People in South Korea between 1992 and 2002. Ph.D. Dissertation, University of Wisconsin, Madison, USA.

(Park and Yu 2011) Park, Byeong-geum and Eun-seon. 2011. A Study on Adolescents' Right Recognition and Self-Esteem. Studies on Adolescents 18: 89-116.

(Nam 2013) Nam, In-woo. 2013. Pilyeonjeok Gyeonghum, Saeroun Changjak Bangsik. Yeongeuksokui Cheongsonyeon, Cheongsonyeonsokui Yeongeuk. Edited by TYA Research Center. Seoul: NTCK, 87-97. 33-45. 979-11-8538902-8. 